\title{
Hysteria, hysterectomy, and anti-NMDA receptor encephalitis: a modern perspective on an infamous chapter in medicine
}

In the online version of this Filler by Thomas A Pollak (BMJ 2013;346:f3756, doi:10.1136/bmj.f3756) there is a mistake in the fourth paragraph. The second sentence should have read: "The weight and influence of centuries of misogynistic theorising by an all-male medical establishment cannot be overestimated [not "underestimated," as published]."

Cite this as: BMJ 2013;347:f4456

๑ BMJ Publishing Group Ltd 2013 